\title{
WHISTLER-MODE CHORUS AND HISS IN THE INNER MAGNETOSPHERE OF EARTH: CONSEQUENCES FOR THE JUICE PROJECT
}

\author{
O. Santolik*†, J. Soucek*, I. Kolmasova*, G. B. Hospodarsky \\ W. S. Kurth ${ }^{\ddagger}$, C. A. Kletzing ${ }^{\ddagger}$, and J.-E. Wahlund ${ }^{\S}$
}

\begin{abstract}
Previous observations have shown the importance of measurements of waves in plasmas around Jovian moons, especially in the light of recent advances in analysis of whistler-mode waves in Earth's magnetosphere and their importance for acceleration of radiation belt electrons to relativistic energies. We analyze whistler-mode chorus and hiss using a database of measurements of the Waves instruments of the Electric and Magnetic Field Instrument Suite and Integrated Science (EMFISIS) onboard the Van Allen Probes in Earth's radiation belts. We estimate the timefrequency structure, polarization and propagation parameters of these waves. We show that high resolution multicomponent measurements of the fluctuating magnetic and electric fields are necessary for proper characterization of hiss and chorus. Proposed measurement modes for the low frequency receiver subsystem of the Radio \& Plasma Waves Investigation (RPWI) experiment, which will be implemented on the JUICE (JUpiter ICy moon Explorer) spacecraft, include onboard processing designed to be suitable for a similar analysis of whistler-mode waves in the magnetospheres of Jupiter and Ganymede.
\end{abstract}

\footnotetext{
*Institute of Atmospheric Physics, Czech Academy of Sciences, Prague, Czechia

${ }^{\dagger}$ Charles University, Prague, Czechia

$¥$ Department of Physics and Astronomy, University of Iowa, Iowa City, IA, USA

$\S$ Swedish Institute of Space Physics, Uppsala, Sweden
} 
